Research article Open Access

\title{
Bonding of articular cartilage using a combination of biochemical degradation and surface cross-linking
}

\author{
Carsten Englert ${ }^{1}$, Torsten Blunk², Rainer Müller ${ }^{3}$, Sabine Schulze von Glasser ${ }^{1}$, Julia Baumer ${ }^{2}$, \\ Johann Fierlbeck ${ }^{4}$, Iris M Heid5,6, Michael Nerlich ${ }^{1}$ and Joachim Hammer ${ }^{4}$
}

\begin{abstract}
1Department of Trauma Surgery, University Medical Centre Regensburg, Franz-Josef-Strauss-Allee, 93053 Regensburg, Germany 2Department of Pharmaceutical Technology, University of Regensburg, Universitätsstrasse, 93053 Regensburg, Germany ${ }^{3}$ Institute of Physical and Theoretical Chemistry, University of Regensburg, Universitätsstrasse, 93053 Regensburg, Germany ${ }^{4}$ Mechanical Engineering Faculty, University of Applied Sciences, Galgenbergstrasse, 93053 Regensburg, Germany ${ }^{5}$ GSF-National Research Centre, Institute of Epidemiology, Ingolstädter Landstrasse, 85674 Neuherberg, Germany 6 Institute of Medical Informatics, Biometry, and Epidemiology, Ludwig-Maximilians-University, Munich, Germany
\end{abstract}

Corresponding author: Carsten Englert, carsten.englert@klinik.uni-regensburg.de

Received: 22 Jan 2007 Revisions requested: 10 Apr 2007 Revisions received: 30 Apr 2007 Accepted: 15 May 2007 Published: 15 May 2007

Arthritis Research \& Therapy 2007, 9:R47 (doi:10.1186/ar2202)

This article is online at: http://arthritis-research.com/content/9/3/R47

(c) 2007 Englert et al.; licensee BioMed Central Ltd.

This is an open access article distributed under the terms of the Creative Commons Attribution License (http://creativecommons.org/licenses/by/2.0), which permits unrestricted use, distribution, and reproduction in any medium, provided the original work is properly cited.

\begin{abstract}
After trauma, articular cartilage often does not heal due to incomplete bonding of the fractured surfaces. In this study we investigated the ability of chemical cross-linkers to facilitate bonding of articular cartilage, either alone or in combination with a pre-treatment with surface-degrading agents. Articular cartilage blocks were harvested from the femoropatellar groove of bovine calves. Two cartilage blocks, either after pre-treatment or without, were assembled in a custom-designed chamber in partial apposition and subjected to cross-linking treatment. Subsequently, bonding of cartilage was measured as adhesive strength, that is, the maximum force at rupture of bonded cartilage blocks divided by the overlap area. In a first approach, bonding was investigated after treatment with cross-linking reagents only, employing glutaraldehyde, 1-ethyl-3diaminopropyl-carbodiimide (EDC)/N-hydroxysuccinimide (NHS), genipin, or transglutaminase. Experiments were conducted with or without compression of the opposing surfaces. Compression during cross-linking strongly enhanced bonding, especially when applying EDC/NHS and glutaraldehyde. Therefore, all further experiments were performed under compressive conditions. Combinations of each of the four cross-linking agents with the degrading pre-

treatments, pepsin, trypsin, and guanidine, led to distinct improvements in bonding compared to the use of cross-linkers alone. The highest values of adhesive strength were achieved employing combinations of pepsin or guanidine with EDC/NHS, and guanidine with glutaraldehyde. The release of extracellular matrix components, that is, glycosaminoglycans and total collagen, from cartilage blocks after pre-treatment was measured, but could not be directly correlated to the determined adhesive strength. Cytotoxicity was determined for all substances employed, that is, surface degrading agents and cross-linkers, using the resazurin assay. Taking the favourable cell vitality after treatment with pepsin and EDC/NHS and the cytotoxic effects of guanidine and glutaraldehyde into account, the combination of pepsin and EDC/NHS appeared to be the most advantageous treatment in this study. In conclusion, bonding of articular cartilage blocks was achieved by chemical fixation of their surface components using cross-linking reagents. Application of compressive forces and prior modulation of surface structures enhanced cartilage bonding significantly. Enzymatic treatment in combination with crosslinkers may represent a promising addition to current techniques for articular cartilage repair.
\end{abstract}

\section{Introduction}

After trauma, articular cartilage often does not heal due to incomplete bonding of the fractured surfaces. The pathophysiological mechanism of articular cartilage integration has been intensively investigated in vitro, showing that integration depends on collagen metabolism [1,2], collagen cross-linking [3], cell vitality [4], and hormonal stimulation [5]. Inhibiting factors have also been described, such as synovial fluid components, which may inhibit the integrative repair by binding to the cracked surface [6], cytokines, which abolish the anabolic 
steroid hormone effect [5], and the synovial fluid flow, which might act at the interface in joint motion to keep the surfaces apart [7].

Based on these findings, therapeutic options for articular cartilage integration have been investigated. Collagen cross-linking has been stimulated over time in vitro [8] or articular cartilage surfaces have been degraded in order to stimulate repair in vitro [9-12] as well as in vivo [13]. Enzymes that were employed for articular cartilage degradation included trypsin $[5,11,13]$, chondroitinase ABC [9], and hyaluronidase with subsequent collagenase treatment [12], resulting in enhanced integrative cartilage repair in vitro. Physical swelling of surface structures by guanidine was also reported to stimulate the integrative repair process [10].

Cartilage can be considered as a composite material consisting of a collagen network and other extracellular matrix components, mainly glycosaminoglycans (GAGs). Collagen and its derivatives have been cross-linked for tissue engineering or biomaterial purposes [14,15]. Glutaraldehyde is the most extensively used reagent for cross-linking primary amino groups, mainly exposed by collagen $[16,17]$. However, it has been reported to elicit cytotoxic effects $[18,19]$. In proteoglycans, amino groups are mainly acetylated and, therefore, not subjected to glutaraldehyde cross-linking. Water-soluble carbodiimides activate carboxylic groups of proteins such as collagen, which results in the formation of amide-type cross-links without any residual reactive groups [20,21]. In addition, carbodiimides were found to cross-link hyaluronic acid molecules by forming ester bonds between hydroxyl and carboxyl groups [22]. The carbodiimide method has been shown to be superior to glutaraldehyde in terms of cyto- and biocompatibility $[19,23]$. Another favourable cross-linker for primary amino groups is the naturally occurring reagent genipin, which has been reported to be significantly less cytotoxic than glutaraldehyde [23,24]. Transglutaminase, an enzyme in mammalian chondrocytes whose expression is strongly correlated with cell differentiation, has also been used as a collagen crosslinking reagent [25] and has been introduced for articular cartilage gluing [26]. Taken together, glutaraldehyde, carbodiimides, genipin, and transglutaminase all cross-link functional groups of extracellular matrix components. Such reagents may, therefore, also be used to cross-link exposed functional groups on a fractured surface of articular cartilage after trauma or transplantation.

The objective of this study was to investigate the initiation of immediate bonding of articular cartilage blocks by means of combining cartilage degradation and cross-linking reagents. In addition, it was investigated whether a considerable compression of the cartilage blocks was necessary to achieve bonding. For all combinations, that is, compression of cartilage blocks and application of surface degrading and cross-linking reagents, specific emphasis was put on the achievable adhesive strength of the bonding interface according to the integration model established by Reindel and colleagues [4].

\section{Materials and methods Cartilage preparation, compression, and bonding}

Within one day after sacrificing of 8- to 12-week-old bovine calves, osteochondral fragments were harvested from the femoropatellar groove, using a reciprocating saw (Stryker Instruments, Kalamazoo, Ml, USA). Blocks of $10 \mathrm{~mm} \times 10 \mathrm{~mm}$ in length and $20 \mathrm{~mm}$ in height were harvested (Figure 1a). A sledge microtome (Microm HM440E, Neuss, Germany) was used to cut the osteochondral fragments into cartilage slices of two precisely defined thicknesses, that is, $0.25 \mathrm{~mm}$ (geometry one (G1)) and $0.3 \mathrm{~mm}$ (geometry two (G2)) (Figure 1b). In all cases, the two top slices were discarded and only the following two underlying slices were used for experiments. These slices were cut into rectangles of $8 \mathrm{~mm} \times 2.5 \mathrm{~mm}$ (Figure 1c). During the entire preparation procedure the specimens were kept moist and free of blood by copious irrigation with cooled PBS.

The integration specimens were assembled by positioning two cartilage rectangles in partial apposition, creating a defined overlap area of $4 \mathrm{~mm} \times 2.5 \mathrm{~mm}$ for bonding. The precise assembly was guaranteed by a custom-made chamber and an additional fixation stamp (Figure 1d) [5]. After fixation (without cartilage blocks) a gap of exactly $0.5 \mathrm{~mm}$ between stamp surface and chamber bottom remained. Thus, when the cartilage samples were inserted into the chamber and fixed by the stamp, for two G1 cartilage blocks almost no compression was acting during bonding, whereas for G2 blocks a defined compressive strain of $17 \%$ of the total thickness was applied (Figure 1e). To determine the creep modulus for both geometries (G1 and G2), the custom-made chamber and the stamp were modified and connected to the test rig (Hegewald and Peschke, Nossen, Germany). Samples were compressed by the stamp and the resulting force relaxation behaviour was analysed by recording the load over time.

\section{Cross-linking}

For bonding, the cartilage blocks within the chamber were placed in a 24-well culture plate and each sample was subjected to one of the following cross-linking agents for $2 \mathrm{~h}$ at room temperature $(750 \mu$ per sample): glutaraldehyde (Roth, Germany) at a concentration of $20 \mathrm{mg} / \mathrm{ml}$, buffered in PBS; 1 ethyl-3-diaminopropyl-carbodiimide (EDC) and N-hydroxysuccinimide (NHS) (Fluka, Neu-Ulm, Germany) at concentrations of $20 \mathrm{mg} / \mathrm{ml}$ and $5 \mathrm{mg} / \mathrm{ml}$, respectively, in morpholinoethanesulfonic acid buffered solution ( $\mathrm{pH} 5.5)$; genipin (WAKO Chemicals, Germany) at a concentration of $5 \mathrm{mg} / \mathrm{ml}$ in PBS; transglutaminase (Ajinomoto Foods, Hamburg, Germany) at a final concentration of $60 \mathrm{U}$ per gram dry weight of cartilage block in $0.01 \mathrm{M}$ acetic acid, adjusted to $\mathrm{pH} 6$ (transglutaminase was applied according to the protocol by Chen and colleagues [27], with the exception that cartilage blocks 
Figure 1

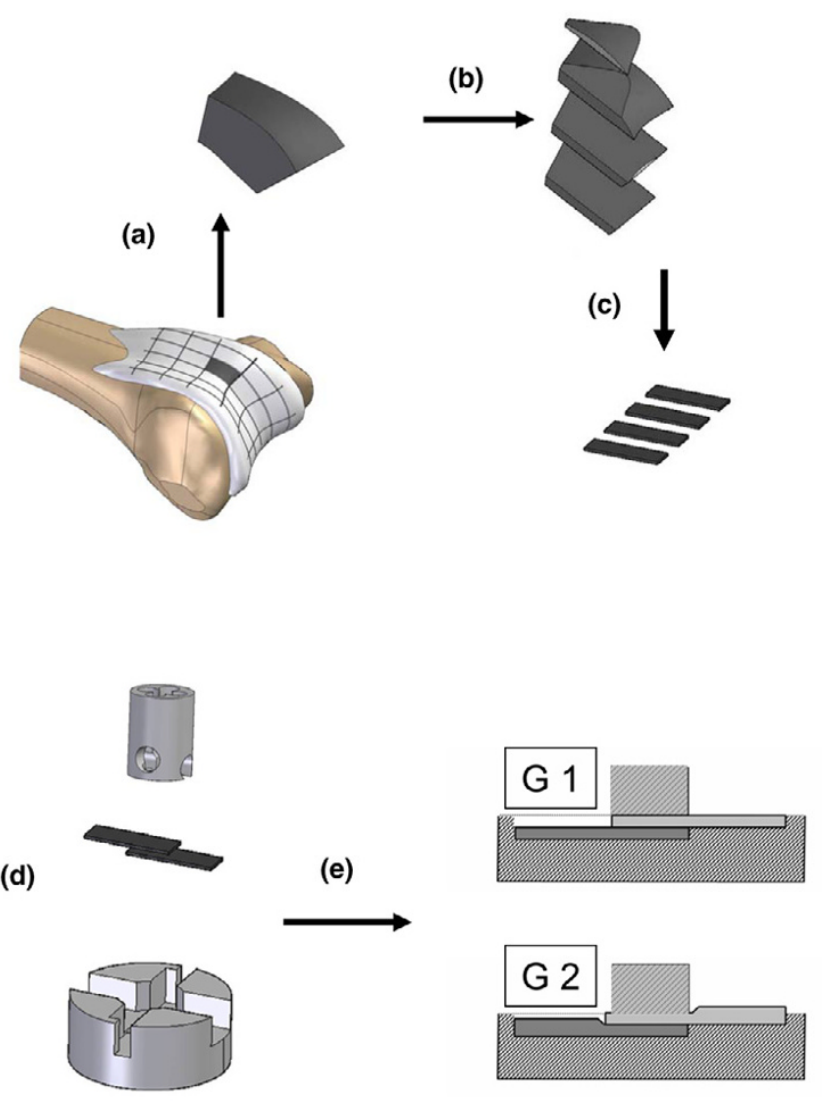

Preparation of the articular cartilage blocks and assembly during bonding experiments. (a) Osteochondral fragments were harvested from the femoropatellar groove of bovine calves. Blocks of $10 \times 10 \times 20 \mathrm{~mm}^{3}$ were harvested. (b) These blocks were cut into cartilage slices of two precisely defined thicknesses, 0.25 and $0.3 \mathrm{~mm}$, which were designated geometry 1 and 2 (G1 and G2) (in the diagram, only one thickness is shown). (c) These slices were cut into rectangles of $8 \mathrm{~mm} \times 2.5$ $\mathrm{mm}$. (d) The integration specimens were assembled by positioning two cartilage rectangles in partial apposition, creating a defined overlap area of $4 \mathrm{~mm} \times 2.5 \mathrm{~mm}$ for bonding. (e) When cartilage samples (either two G1 or two G2 blocks) were inserted into the chamber and fixed by the stamp, different compressive strains were applied.

were incubated in treatment solution for $2 \mathrm{~h}$ in contrast to the described protocol with a treatment duration of $12 \mathrm{~h}$ ). See also Figure 2 for chemical reaction schemes.

\section{Surface degradation}

For improved cross-linking, additional surface degradation treatments prior to cross-linking were applied. In all cases, the tissue samples, that is, the single cartilage pieces, were incubated in a $100 \mu \mathrm{l}$ solution. After surface degradation, all samples were washed three times with PBS before being inserted into the chamber described above for bonding experiments. The following three solutions were used for degradation: trypsin (Gibco, Eggenstein, Germany) at a concentration of $0.5 \mu \mathrm{g} / \mathrm{ml}$ in PBS, pH 7.4 for 30 minutes at room temperature [6]; pepsin from hog stomach with 3,348 U/mg (Sigma-Fluka,
Steinheim Germany), used at $0.5 \mathrm{mg} / \mathrm{ml}$ in PBS for $30 \mathrm{~min}$ utes, $\mathrm{pH} 7.4$ at room temperature; guanidinium hydrochloride (Sigma, Steinheim Germany) at a concentration of $4 \mathrm{~mol} / \mathrm{l}$ for 10 minutes at $10^{\circ} \mathrm{C}$ in a solution that was prepared with sodium acetate and adjusted to $\mathrm{pH} 6.0$ by hydrochloric acid [10].

\section{Biomechanical testing}

The adhesive shear strength after cross-linking was investigated under uniaxial tensile loading, as first described by Reindel and colleagues [4]. All experiments were performed until rupture. Prior to mechanical testing the integrated interface area of each sample was determined by optical microscopy using the imaging software analySIS 3.1 (SZX12, Olympus, Hamburg, Germany). The samples were carefully removed from the incubation chambers and mounted into the fixings of the test rig (Hegewald and Peschke). Particular care was taken to exclude any influence resulting from misalignment in the orientation of the load axis to the neutral fibre of the interface area by using a biaxial positioning device with an accuracy of $0.01 \mathrm{~mm}$. Both custom-designed fixings were equipped with a small vacuum drill hole for accurate adjustment. The final fixing of the samples was achieved by springloaded jaws. The gauge length (that is, free distance between the fixings) was $7 \mathrm{~mm}$ in all cases.

All tests were run at an extension rate of $0.5 \mathrm{~mm} /$ minute. The displacement was continuously measured as the increase in distance between the two fixings by means of a linear variable differential transformer with an accuracy of $0.01 \mathrm{~mm}$ (HBM, Inc., Marlborough, MA, USA; WA/10 mm). The load was recorded using a $100 \mathrm{~N}$ load cell, which was limited to $5 \mathrm{~N}$ effective range (HBM, Inc.; H2/100 N). The accuracy was in the order of $0.01 \mathrm{~N}$. The displacement and the load signal were digitized using a data acquisition card (PCl-MIO-16E-4, National Instruments, Munich, Germany), yielding an accuracy of $0.08 \mathrm{~N}$ for the load signal and $0.06 \mathrm{~mm}$ for the strain signal. The sampling rate of the data was $10 \mathrm{~Hz}$.

Adhesive strength was determined as the maximum shear force at rupture divided by the measured overlap area. Samples that failed to adhere, which became obvious during removal from the culture chamber or during placement into clamps, were assigned an adhesive strength of $0 \mathrm{kPa}$.

\section{Determination of glycosaminoglycan and collagen content}

To assess the effects of the surface degradation treatment, the extracellular matrix content of cartilage blocks was analysed after being subjected to the respective agents. Additionally, the supernatant was analysed for released extracellular matrix components. Before analysis, cartilage samples were digested with $1 \mathrm{ml}$ of a papainase solution $(3.2 \mathrm{U} / \mathrm{ml}$ in buffer) for $18 \mathrm{~h}$ at $60^{\circ} \mathrm{C}$. Sulfated GAG content was determined spectrophotometrically at $525 \mathrm{~nm}$ after the reaction with 
(a)

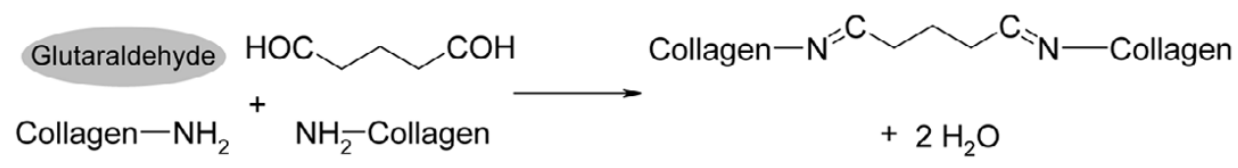

(b)

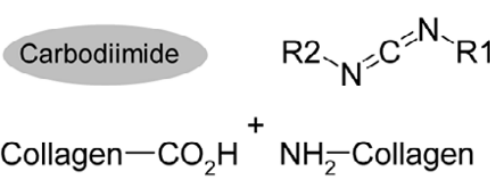

(c)

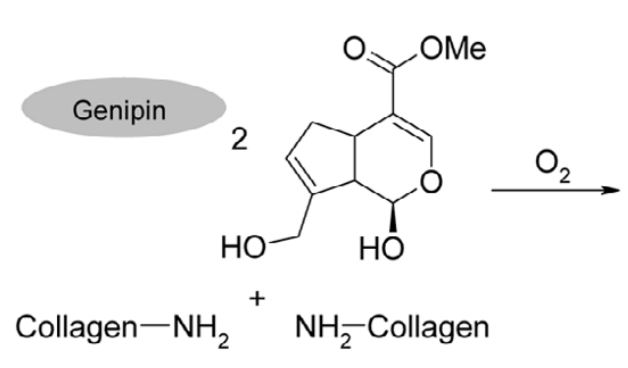

(d)

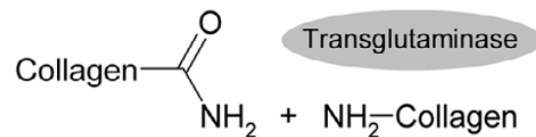<smiles>[R2]NC(=O)NC(=O)C1CCCCC1</smiles>

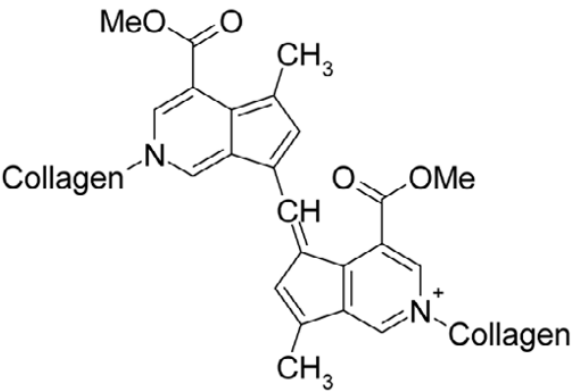

(1)

.

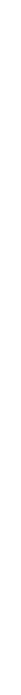

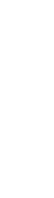


Figure 3

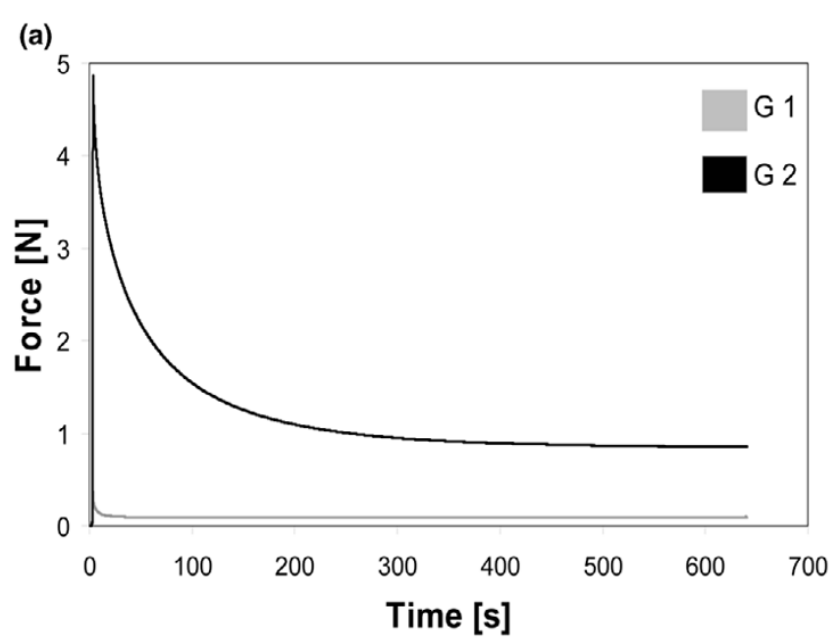

(b)

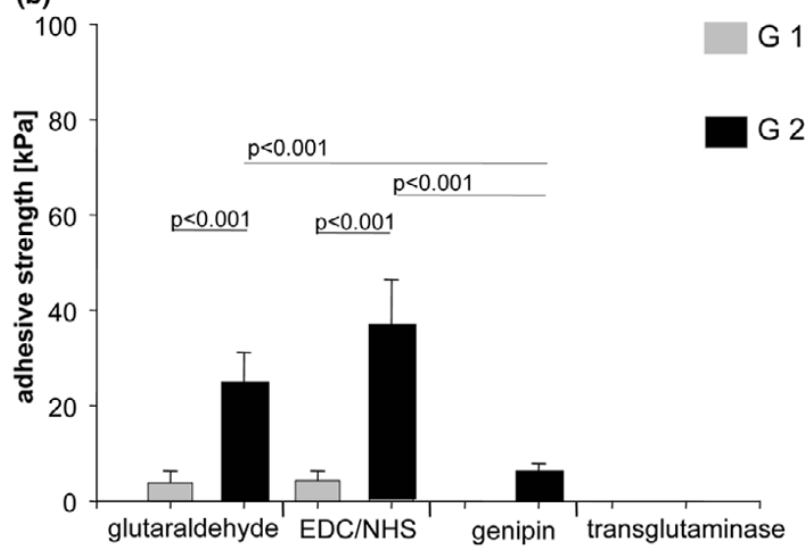

Stress-relaxation curves for the two sample geometries and bonding dependence on compression. (a) Stress-relaxation curves for the sample geometries G1 and G2 (see Figure 1e) were determined in a standardised creep modulus set-up. Samples were compressed by a stamp and the resulting force relaxation behaviour was analysed by recording the load over time. (b) G1 (almost no compression) or G2 (compression) cartilage blocks were subjected to different cross-linkers (without degrading pre-treatment). Adhesive strength as a measure of bonding was determined immediately after cross-linking. Bars represent the mean with standard error of the mean of at least 16 samples derived from 4 independent experiments, each with at least 4 replicates per group. $P$ values in the graph are from pairwise comparisons using the Mann Whitney-U test. EDC, 1-ethyl-3-diaminopropyl-carbodiimide; $\mathrm{NHS}, \mathrm{N}$-hydroxysuccinimide.

cross-linking reagents, the same concentrations as stated in the cross-linking and surface degradation sections were used (see above).

\section{Statistical analysis}

The statistical analysis was carried out using SPSS $12.0 \mathrm{G}$ (SPSS, Munich, Germany). Of the free analysed parameters, adhesive strength $(\mathrm{kPa}), \mathrm{GAG}$ content $(\mu \mathrm{g})$, collagen content $(\mu \mathrm{g})$, and relative fluorescence unit (RFU), the Kolmogorov-
Smirnov test showed evidence against normal distribution for adhesive strength. Thus, the non-parametric Kruskal Wallis test for overall testing and the Mann-Whitney-U test for pairwise testing was applied to test for differences in adhesive strength between groups. For analysis of the GAG and collagen content of the cartilage blocks or cytotoxicity (RFU) of the applied agents, which were normally distributed, overall difference in the groups was assessed by analysis of variance (ANOVA) followed by post hoc comparisons made by Tukey's test. Throughout, statistical significance was accepted for $p<$ 0.05 .

\section{Results}

Adhesive strength of the bonding area

At first, cartilage blocks of the two different geometries were fixed in partial apposition in the custom-made chamber. The relaxation behaviour of the $\mathrm{G} 1$ and $\mathrm{G} 2$ cartilage blocks is shown in Figure 3a. Due to the precise positioning of the G1 cartilage blocks in the chamber, immediately after mechanical fixation an instantaneous load drop to almost $0 \mathrm{~N}$ was observed. The remaining compressive load, in the order of 0.1 $\mathrm{N}$, was attributed to swelling of the cartilage. In contrast, for the oversized G2 cartilage blocks, a compressive load of $5 \mathrm{~N}$ resulted from mechanical fixing by the stamp. With increasing incubation time and force relaxation, the load decreased and approached $1 \mathrm{~N}$ after approximately $400 \mathrm{~s}$.

To compare the two geometries $\mathrm{G} 1$ and $\mathrm{G} 2$ with regard to cartilage bonding, the cross-linking agents were applied without prior surface degradation. The Kruskal Wallis test showed significant difference in adhesive strength $(\mathrm{kPa})$ between the groups, which motivated us to perform pairwise testing. Compression to $83 \%$ of initial thickness during incubation (G2) resulted in strongly enhanced bonding after treatment with glutaraldehyde and EDC/NHS compared to no compression (Figure 3b). Using transglutaminase, no bonding occurred without prior surface degradation for either of the two geometries. Based on these results, the following experiments investigating the effects of the different combinations of degrading and cross-linking agents were conducted using G2 cartilage blocks. It should be noted that in experiments with neither surface degradation nor cross-linking or in experiments with surface degradation only, no bonding, for either G1 or G2, was achieved at all.

The four cross-linking reagents glutaraldehyde, genipin, EDC/ $\mathrm{NHS}$, and transglutaminase were each combined with the pretreatments trypsin, pepsin, and guanidine; the resulting bonding quality measured as adhesive strength is shown in Figure 4. The Kruskal Wallis test showed significant differences in adhesive strength $(\mathrm{kPa})$ between the groups, prompting us to perform pairwise testing. With glutaraldehyde, only guanidine pre-treatment led to a significant increase of adhesive strength (55 kPa compared to $20 \mathrm{kPa}$ for the group with no pre-treatment; Figure 4a). In combination with EDC/NHS, pepsin or 
Figure 4

(a)

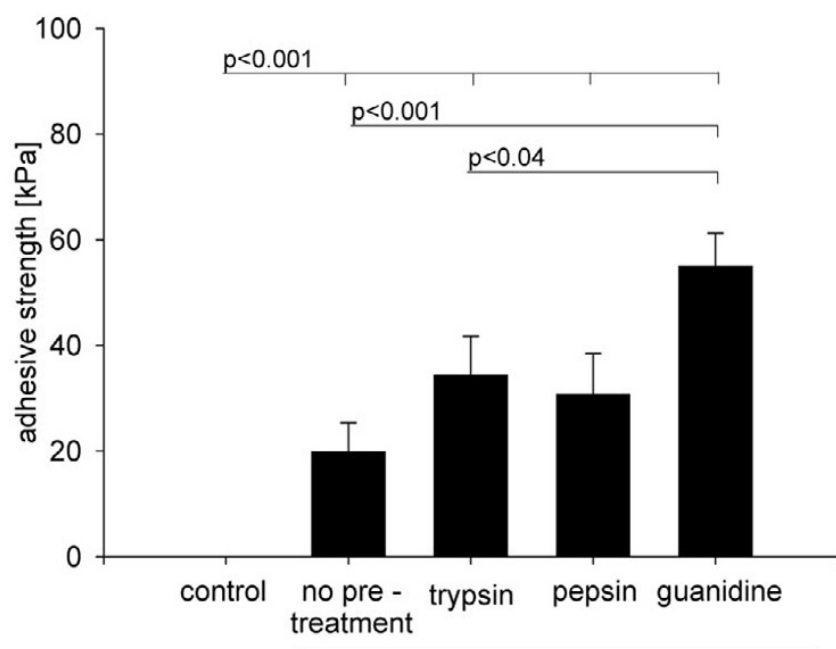

(c)

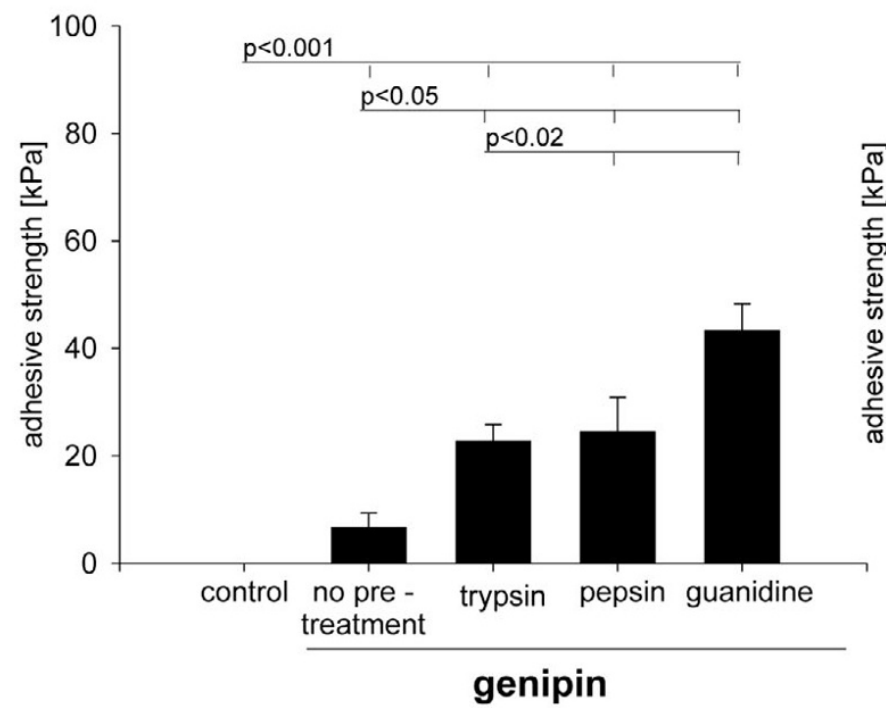

(b)

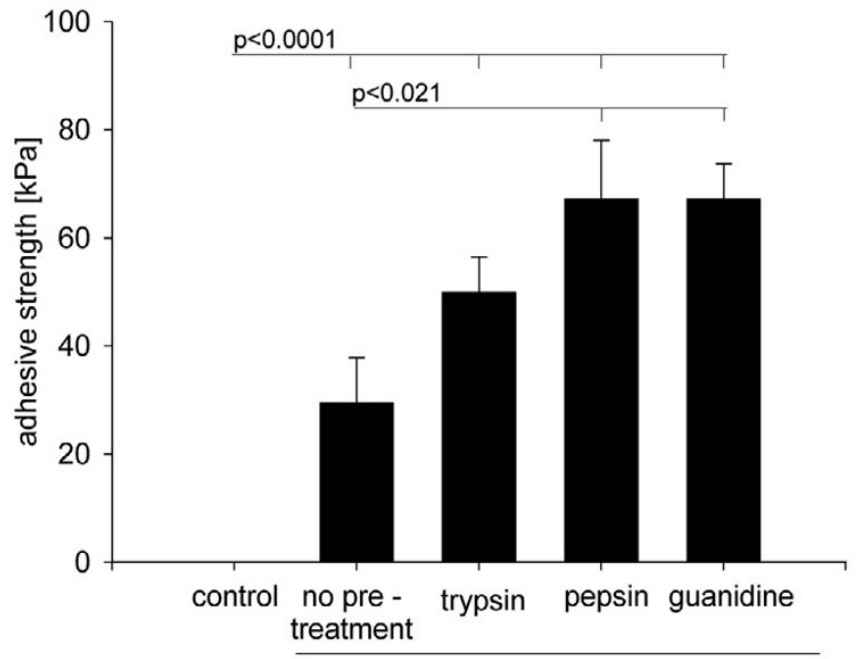

(d)

EDC/NHS

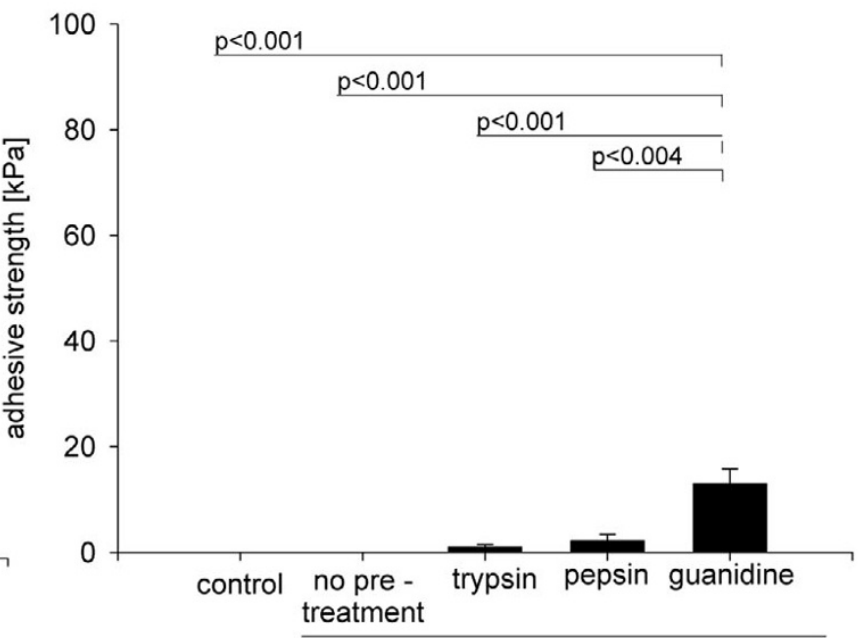

transglutaminase

Bonding of cartilage blocks after treatment with surface-degrading reagents and subsequent cross-linking. Adhesive strength as a measure of bonding was determined immediately after cross-linking with (a) glutaraldehyde, (b) 1-Ethyl-3-diaminopropyl-carbodiimide (EDC)/N-hydroxysuccinimide (NHS), (c) genipin, or (d) transglutaminase. Before cross-linking, cartilage blocks were pre-treated with either trypsin, pepsin, or guanidine, or blocks were cross-linked without pre-treatment ('no pre-treatment'). In the control group, neither pre-treatment nor cross-linking were performed. Bars represent the mean with standard error of the mean of 20 samples derived from 4 independent experiments, each with 5 replicates per group. $P$ values are from Mann Whitney-U test for pairwise comparisons.

guanidine pre-treatment increased the adhesive strength to 65 $\mathrm{kPa}$, exhibiting the highest values seen among all combinations of pre-treatment and cross-linking reagents in this study (30 kPa for no pre-treatment; Figure 4b). With genipin, all three pre-treatments led to a significantly increased adhesive strength, with the highest values detected with guanidine; however, mean values were all below those for EDC/NHS and glutaraldehyde (Figure 4c). For transglutaminase cross-linking, pre-treatment with guanidine was necessary to induce notice- able bonding. Overall, transglutaminase clearly resulted in the lowest values for adhesive strength compared to all other cross-linkers (Figure 4d).

\section{Effects of surface degradation on glycosaminoglycan and collagen content}

To determine the effects of the degrading agents on extracellular matrix content, the GAG and total collagen content were determined in cartilage samples and the respective superna- 
Figure 5

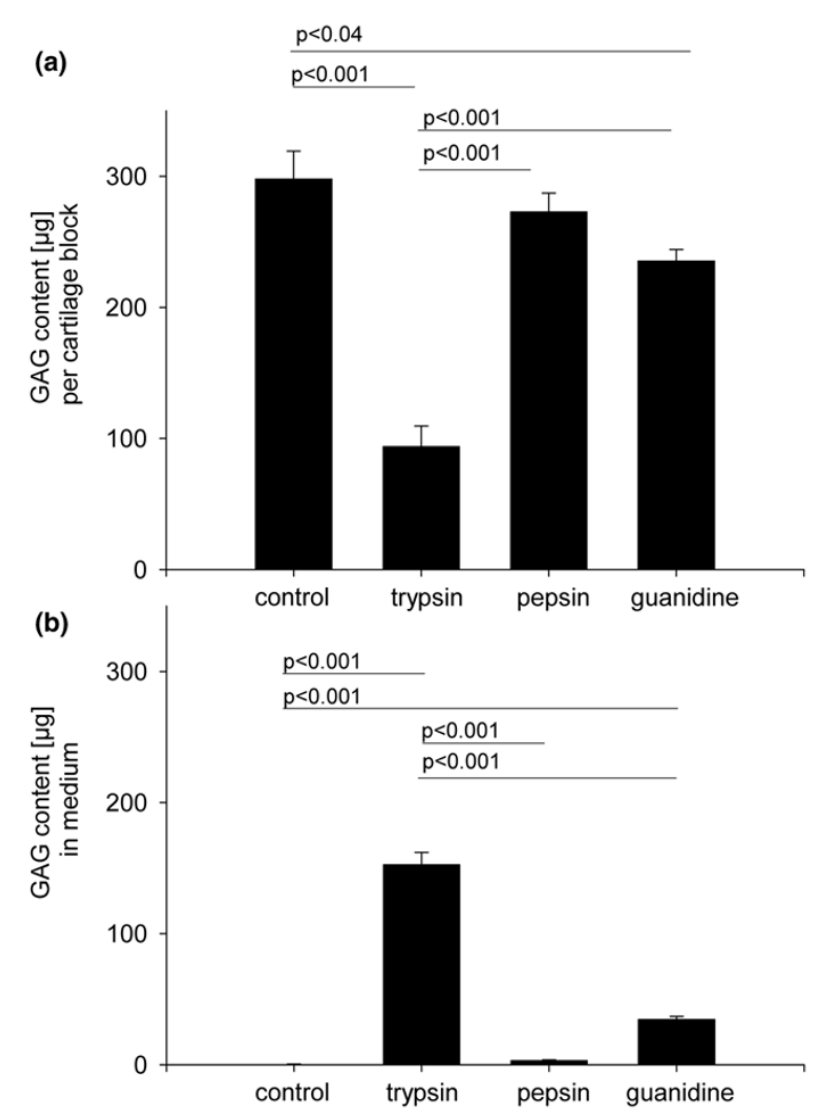

(c)

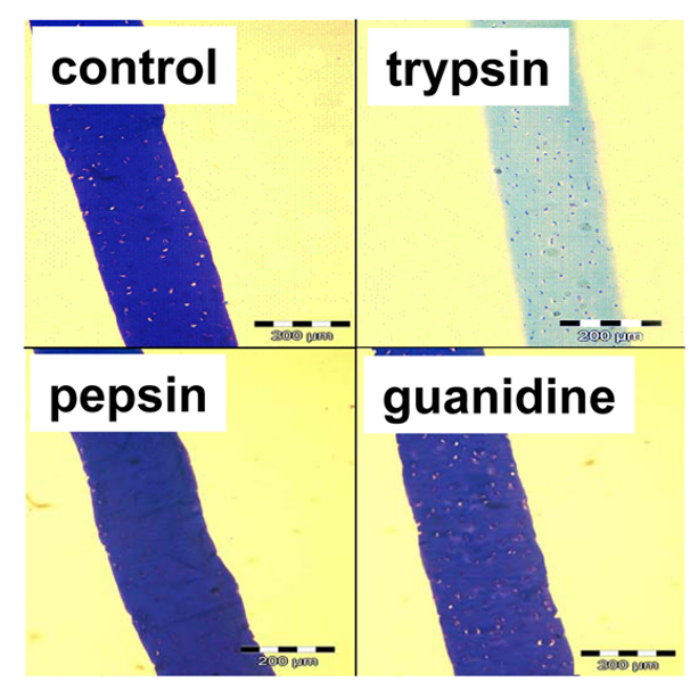

Glycosaminoglycan (GAG) release from cartilage blocks determined after treatment with surface-degrading agents. Cartilage blocks were subjected to pre-treatment with trypsin, pepsin, or guanidine, as indicated for the bonding experiments; the samples in the control group were incubated in PBS buffer. (a) Subsequently, the GAG content within the cartilage blocks was determined. (b) Additionally, the amount of GAG released into the medium (per cartilage block) was measured. Nine samples were measured per group. Bars represent the mean with standard error of the mean. $P$ values are from post hoc Tukey test for pairwise comparisons. (c) Additionally, histological cross-sections of cartilage blocks were stained for GAGs. tants after treatment. The ANOVA test showed significant differences between the groups $(p<0.001)$. Trypsin treatment strongly decreased the GAG content of cartilage samples to $31 \%$ of the control group (Figure $5 \mathrm{a}$ ); whereas untreated control blocks had a GAG content of $4.2 \%$ per wet weight, trypsin reduced the GAG content to 1.3\%. Guanidine treatment resulted in a reduction in GAG content to $78 \%$ of that of the control group, whereas only small amounts of GAG were released from the cartilage samples treated with pepsin (Figure $5 a$ ). The GAG release from cartilage samples was confirmed by analysis of the corresponding supernatants (Figure $5 b)$ and staining of histological cross-sections of cartilage blocks with toluidine blue for GAG (Figure 5c). The collagen content of untreated control blocks was $12.8 \%$ per wet weight. For all three treatments, trypsin, pepsin, or guanidine, no significant reduction in collagen content in the cartilage samples was detected.

\section{Relative cytotoxicity}

The resazurin cytotoxicity test performed on cartilage samples revealed distinct differences for the reagents employed in this study ( $p$ value from ANOVA, $p<0.001$ ). Among the degrading reagents, pepsin had only small cytotoxic effects whereas guanidine exhibited strong effects in comparison to the nontreated control group (Figure 6a). In the cross-linking reagent group, EDC/NHS showed almost no effect, whereas reduced metabolic activity to less than $50 \%$ within $2 \mathrm{~h}$ was detected for glutaraldehyde and genipin (Figure 6b).

\section{Discussion}

In this study, the effects of compression of the cartilage interface, surface degradation, and biochemical cross-linking on articular cartilage bonding were investigated. Specific emphasis was put on the resulting mechanical stability of the bonded interface due to molecular bridging of opposing surface structures. This immediate repair technique might provide one further option for the therapeutic treatment of articular cartilage wounds.

\section{Compression}

In the absence of treatment with cross-linking agents, neither just laying the cartilage blocks together nor compressing them (to $83 \%$ of initial thickness) had any effect on bonding between them. Although the cross-linking reagents EDC/NHS and glutaraldehyde led to measurable bonding of cartilage blocks in the absence of compressive strain, large increases in adhesive strength were achieved by additional compressive load during the bonding procedure. Therefore, all experiments investigating combinations of cross-linking reagents with a pre-treatment with surface degrading agents were carried out under compressive load conditions.

\section{Cross-linking}

EDC/NHS can non-specifically catalyse covalent binding of the amino or carboxyl groups of collagen; furthermore, carbox- 
Figure 6

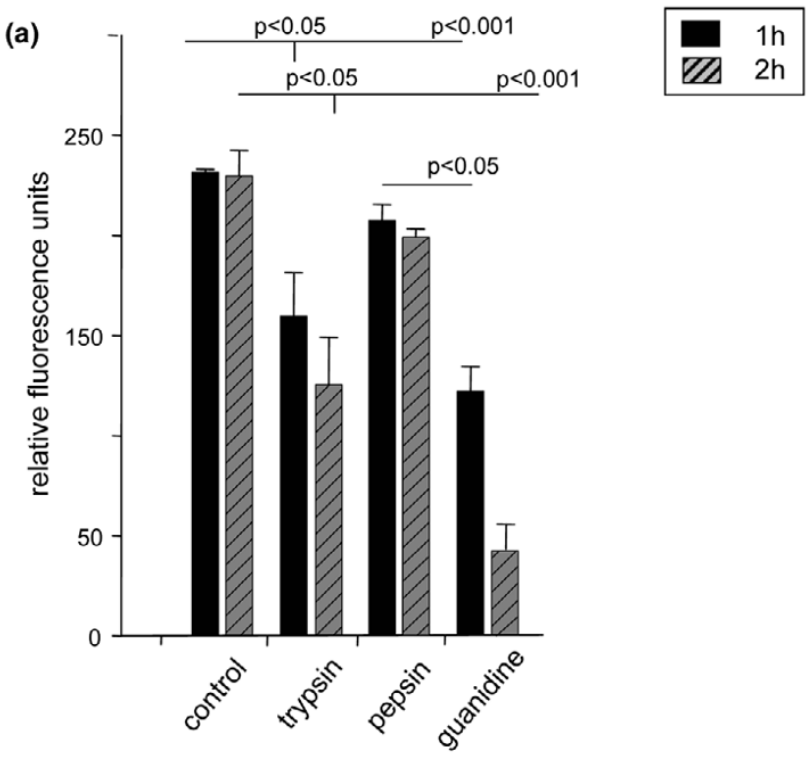

(b)

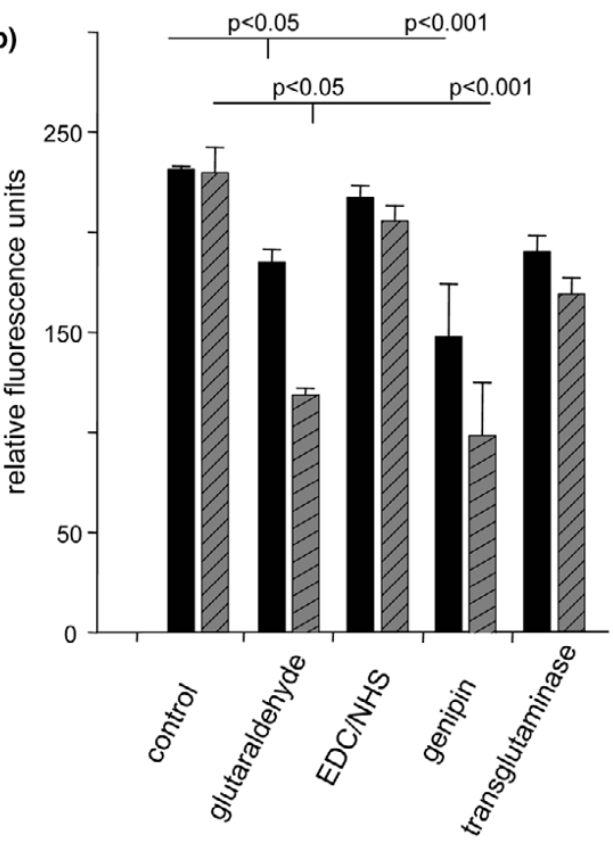

Cytotoxicity of degrading and cross-linking agents. The relative cytotoxicity of (a) degrading and (b) cross-linking agents was determined using the resazurin assay (expressed as relative fluorescence units). Cartilage blocks were treated with the respective reagents for either one or two hours. The controls were incubated in PBS. $P$ values are from Tukey test for pairwise comparisons. EDC, 1-ethyl-3-diaminopropyl-carbodiimide; NHS, N-hydroxysuccinimide.

ylic groups of GAGs may also be involved. In our study, EDC/ NHS was the best cross-linking reagent with regard to bonding of articular cartilage blocks in all investigations, that is, when comparing the cross-linkers alone and in combination with degrading pre-treatment. The combinations of EDC/NHS with pepsin or guanidine pre-treatment led to the highest adhesive strengths detected in this study. To date, cell-based articular cartilage repair in vitro has been correlated with cell metabolism [2], collagen deposition [1] and collagen crosslinking [3,31]. However, the impressive results yielded with EDC/NHS, the only cross-linker in this study able to additionally catalyse binding of GAGs, may support the idea that molecules other than those involved in the collagen network can also contribute to the integrative process in vitro.

With regard to cytoxicity, even after exposure for two hours, EDC/NHS elicited no significant effects, whereas glutaraldehyde and genipin compromised cell vitality considerably. In previous investigations, EDC/NHS has also been observed to be advantageous in this respect, compared to glutaraldehyde [32]. Furthermore, the presented adhesive strength data resulted from a total incubation with cross-linking reagents for two hours. In additional experiments in which guanidine or pepsin pre-treated samples were exposed to EDC/NHS for only 30 or 10 minutes, an adhesive strength between 58 and $56 \mathrm{kPa}$ was observed, that is, there was no significant difference to the prolonged treatment of two hours. An exposure for only 10 minutes would further reduce the risk of any cytotoxic effects.

Glutaraldehyde and genipin cross-link amino groups of proteins; glutaraldehyde molecules can cross-link to each other and the chain building properties may have beneficial effects in comparison to genipin, which can only cross-link in pairs [33]. In this study, glutaraldehyde alone yielded higher adhesive strengths than genipin alone and also had slight advantages in combination with pre-treatments. With regard to cytotoxicity, a better tolerance for genipin in comparison to glutaraldehyde has been shown in other investigations utilising 3T3 mouse fibroblasts [34], human osteoblasts [35] and a subcutaneous chamber in mice [36]. In our study, both agents exhibited similarly strong cytotoxic effects.

Transglutaminase, a naturally occurring enzyme in articular cartilage, catalyses a specific collagen cross-linking reaction between lysine and glutamine residues. This enzyme has been previously introduced, combined with compressive load, to enhance integrative bonding of articular cartilage wounds [26]. In our investigation, bonding was detectable only in combination with guanidine pre-treatment; however, compared to the other cross-linking options investigated in this study, transglutaminase resulted in rather weak bonding. The protocol employed in this study was initially described by Chen and colleagues [27] for cross-linking collagen matrices and may not be well transferable to articular cartilage. The reduced reaction time in this study $(2 \mathrm{~h})$ compared to that reported previously (12 h) may also have contributed to the reduced effect. Nevertheless, transglutaminase may still play an important role in in vitro and in vivo integrative repair. Transglutaminase has been previously shown to be biocompatible [37], which was also 
found in this study, with no significant differences to the control. This enzyme, with its specific catalysing mechanism, may be especially beneficial in an ongoing integrative repair process in which newly synthesised collagen fibrils are present, in contrast to a static experimental setting such as used in this study.

For many years, soft tissue adhesives like fibrin [38] have been used for cartilage repair or as an additive in autologous chondrocyte transplantation $[39,40]$. They have been found to be supportive in chondrocyte transplantation or to seal the periosteum flap to the cartilage in vitro, but in vivo fibrin glue did not provide enough mechanical strength to hold the periosteum flap in place [41]. As a further alternative, several synthetic materials have been employed as glues for soft tissues, for example, aminopropyltrimethoxysilane-methylenebisacrylamide siloxane or n-butylcyanoacrylate [42]. In general, the bonding mechanisms of these polymeric substances differ from those of the chemical reagents used in the present work. The polymers penetrate the soft tissue to a certain extent and adhesion is achieved through an interpenetrating network that is irremovable and may impair tissue development at the integration site. In contrast, the chemical cross-linker induces formation of covalent bonds on the surface of the soft tissue. In our opinion, EDC/NHS may be beneficial compared to polymer glue and other chemical cross-linkers (glutaraldehyde and genipin) due to its pure catalysing function. EDC/NHS will not be incorporated into the cartilage and can be easily removed and the scar tissue can be remodelled by cell and extracellular matrix turnover.

\section{Degradation}

Degradation or swelling of articular cartilage surfaces have been reported to be beneficial in cell-based integrative repair in vitro [6,9-12]. In our study, bonding between cartilage blocks did not occur by merely treating the blocks with trypsin, pepsin, or guanidine (even under compressive conditions). However, pre-treatment with the endopeptidases pepsin or trypsin before cross-linking led to distinct improvements in bonding compared to the use of cross-linkers alone. This was particularly the case for the combination of pepsin with EDC/ NHS, for which high values for adhesive strength were achieved. With regard to cytoxicity, pepsin led to no significant effects, whereas trypsin treatment compromised cell vitality considerably.

Pre-treatment with guanidine led to the highest adhesive strengths in combination with all cross-linkers compared to the endopeptidase pre-treatments. Unfortunately, guanidine elicited the strongest cytotoxic effects of all reagents in the study. It is noteworthy that the achieved mechanical bonding is comparable to previous studies employing a similar model. Reindel and colleagues [4] first reported a mechanical adhesive strength of $34 \mathrm{kPa}$ in integrative experiments. Subsequently, studies including degradation with trypsin followed by cultivation reported enhanced adhesive strengths up to 100 $\mathrm{kPa}$ [6]. In the present study, adhesive strengths up to $65 \mathrm{kPa}$ were achieved by guanidine or pepsin pre-treatment and EDC/NHS cross-linking.

Previously, it was assumed that degrading surface treatment led to cell proliferation or stimulation of cell metabolism [12]. The observation from our investigations that cross-linking reagents lead to significantly stronger bonding of cartilage blocks after degradation or swelling pre-treatment implies another hypothesis. The accessibility of functional groups is enhanced by both treatments and, therefore, may have led to a better bonding in our study and better integrative repair in previously described studies. It remains to be clarified on which components of the extracellular matrix these functional groups are located. The endopeptidase trypsin was clearly the most effective at releasing GAGs from the cartilage blocks, whereas pepsin released only a minor fraction of the GAGs. The treatment of cartilage blocks with guanidine prior to biochemical cross-linking leads, in theory, primarily to reduction of non-covalent bonding between molecules of the extracellular matrix. The tertiary structure of matrix molecules, especially GAGs, becomes more open after hydrogen bonds are broken. Additionally, elevated water uptake may occur due to more accessible functional groups or a loosened collagen network with increased pore diameter (swelling). Nevertheless, in our study, guanidine also led to the release of a significant fraction of GAGs from the cartilage blocks. As trypsin led to the lowest adhesive strength values of all surface-degrading agents in this study, the bonding observed can not be directly correlated with the amounts of GAGs released. On the contrary, the large amount of GAGs released by trypsin may have compromised the cartilage structure. For total collagen, no significant release was detected for all the pre-treatments. However, only small changes in the structure of the cartilage surface, which are triggered by the pre-treatment agents, but which are not detectable by the assays employed in this study, may be necessary to elicit distinctly improved responses to the cross-linkers.

Clarification of the mechanisms involved appears to be a worthwhile subject for further investigation. Future studies should also address the fact that immature and mature cartilage differ in extracellular matrix content, structure and mechanical properties [43-45]. In aging, cartilage undergoes structural changes that affect the susceptibility to degradation [46-48]. It is also known that integrative bonding is influenced by the developmental stage of articular cartilage [4]. Therefore, in future studies, the introduced treatment may have to be adjusted to adult cartilage. Furthermore, it has to be noted that for clinical applications special care should be taken to limit any treatment with degrading and cross-linking agents to the area close to the cartilage wound surface. In addition, cell culture experiments after bonding should assure the long-term viability of the treated cartilage. 


\section{Conclusion}

This study clearly demonstrates that immediate bonding of articular cartilage blocks can be achieved by means of chemical cross-linking. Adhesive strength was superior under compressive conditions compared to no compression. In general, pre-treatment with surface-degrading enzymes or swelling by guanidine salt led to distinct enhancement of cartilage bonding after chemical cross-linking. Taking both the observed bonding and the cell vitality after treatment into account, the combination of pepsin pre-treatment and crosslinking with EDC/NHS appears to be the most favourable with regard to this study. The presented work suggests that a combination of selected surface-degrading agents and chemical cross-linkers is a promising option for enhancing bonding of opposed surfaces in cartilage repair.

\section{Competing interests}

The authors declare that they have no competing interests.

\section{Authors' contributions}

CE conceived of the study and its design, participated in the bonding experiments, and helped to draft the manuscript. TB participated in the design of the study and drafted the manuscript. RM participated in the design of the study, specifically with regard to chemical cross-linking. SSvG carried out the bonding experiments and the cytotoxicity assay. JB carried out the analysis of the extracellular matrix content. JF carried out the analysis of the relaxation behaviour and participated in the bonding experiments. IH helped to perform the statistical analysis. $\mathrm{MN}$ and $\mathrm{JH}$ participated in the design and coordination of the study. All authors read and approved the final manuscript.

\section{Acknowledgements}

The authors thank Richard Kujat, $\mathrm{PhD}$, and Tom Böttner for laboratory assistance. The authors thank, in particular, Metzgerei Stierstorfer, Wenzenbach, for the kind supply of bovine joints two hours after sacrificing. One of the authors (CE) is grateful to the University Medical Centre Regensburg for financial support (ReForM A).

\section{References}

1. DiMicco MA, Sah RL: Integrative cartilage repair: adhesive strength is correlated with collagen deposition. J Orthop Res 2001, 19:1105-1112.

2. DiMicco MA, Waters SN, Akeson WH, Sah RL: Integrative articular cartilage repair: dependence on developmental stage and collagen metabolism. Osteoarthritis Cartilage 2002, 10:218-225.

3. Ahsan T, Lottman LM, Harwood F, Amiel D, Sah RL: Integrative cartilage repair: inhibition by beta-aminopropionitrile. J Orthop Res 1999, 17:850-857.

4. Reindel ES, Ayroso AM, Chen AC, Chun DM, Schinagl RM, Sah $\mathrm{RL}$ : Integrative repair of articular cartilage in vitro: adhesive strength of the interface region. J Orthop Res 1995, 13:751-760.

5. Englert C, Blunk T, Fierlbeck J, Kaiser J, Stosiek W, Angele P, Hammer J, Straub R: Steroid hormones strongly support articular cartilage integration in the absence of interleukin-1 $\beta$. Arthritis Rheum 2006, 54:3890-3897.

6. Englert C, McGowan KB, Klein TJ, Giurea A, Schumacher BL, Sah $\mathrm{RL}$ : Inhibition of integrative cartilage repair by proteoglycan 4 in synovial fluid. Arthritis Rheum 2005, 52:1091-1099.
7. Ahsan T, Sah RL: Biomechanics of integrative cartilage repair. Osteoarthritis Cartilage 1999, 7:29-40.

8. McGowan KB, Sah RL: Treatment of cartilage with beta-aminopropionitrile accelerates subsequent collagen maturation and modulates integrative repair. J Orthop Res 2005, 23:594-601.

9. Lee MC, Sung KL, Kurtis MS, Akeson WH, Sah RL: Adhesive force of chondrocytes to cartilage. Effects of chondroitinase ABC. Clin Orthop 2000, 370:286-294.

10. Giurea A, DiMicco MA, Akeson WH, Sah RL: Development-associated differences in integrative cartilage repair: roles of biosynthesis and matrix. J Orthop Res 2002, 20:1274-1281.

11. Obradovic B, Martin I, Padera RF, Treppo S, Freed LE, VunjakNovakovic G: Integration of engineered cartilage. J Orthop Res 2001, 19:1089-1097.

12. van de Breevaart $B J$, In der Maur $C D$, Bos PK, Feenstra $L$, Verhaar $J A$, Weinans $H$, van Osch GJ: Improved cartilage integration and interfacial strength after enzymatic treatment in a cartilage transplantation model. Arthritis Res Ther 2004, 6:R469-R476.

13. Hunziker EB, Rosenberg LC: Repair of partial-thickness defects in articular cartilage: cell recruitment from the synovial membrane. J Bone Joint Surg Am 1996, 78:721-733.

14. Angele $P$, Abke J, Kujat $R$, Faltermeier $H$, Schumann $D$, Nerlich $M$, Kinner B, Englert C, Ruszczak Z, Mehrl R, et al:: Influence of different collagen species on physico-chemical properties of crosslinked collagen matrices. Biomaterials 2004, 25:2831-2841.

15. Bigi A, Burghammer M, Falconi R, Koch MH, Panzavolta S, Riekel C: Twisted plywood pattern of collagen fibrils in teleost scales: an X-ray diffraction investigation. J Struct Biol 2001 136:137-143.

16. Ruijgrok JM, de Wijn JR, Boon ME: Glutaraldehyde crosslinking of collagen: effects of time, temperature, concentration and presoaking as measured by shrinkage temperature. Clin Mater 1994, 17:23-27.

17. Simionescu A, Simionescu D, Deac R: Lysine-enhanced glutaraldehyde crosslinking of collagenous biomaterials. J Biomed Mater Res 1991, 25:1495-1505.

18. van Luyn MJ, van Wachem PB, Damink LO, Dijkstra PJ, Feijen J, Nieuwenhuis $P$ : Relations between in vitro cytotoxicity and crosslinked dermal sheep collagens. J Biomed Mater Res 1992, 26:1091-1110.

19. van Wachem PB, van Luyn MJ, Olde Damink LH, Dijkstra PJ, Feijen J, Nieuwenhuis P: Biocompatibility and tissue regenerating capacity of crosslinked dermal sheep collagen. J Biomed Mater Res 1994, 28:353-363.

20. Olde Damink LH, Dijkstra PJ, van Luyn MJ, van Wachem PB, Nieuwenhuis $\mathrm{P}$, Feijen J: In vitro degradation of dermal sheep collagen cross-linked using a water-soluble carbodiimide. Biomaterials 1996, 17:679-684.

21. Olde Damink LH, Dijkstra PJ, van Luyn MJ, van Wachem PB, Nieuwenhuis $\mathrm{P}$, Feijen J: Cross-linking of dermal sheep collagen using a water-soluble carbodiimide. Biomaterials 1996, 17:765-773.

22. Tomihata $\mathrm{K}$, Ikada $\mathrm{Y}$ : Crosslinking of hyaluronic acid with watersoluble carbodiimide. J Biomed Mater Res 1997, 37:243-251.

23. Chang Y, Tsai CC, Liang HC, Sung HW: Reconstruction of the right ventricular outflow tract with a bovine jugular vein graft fixed with a naturally occurring crosslinking agent (genipin) in a canine model. J Thorac Cardiovasc Surg 2001, 122:1208-1218.

24. Sung HW, Huang RN, Huang LL, Tsai CC: In vitro evaluation of cytotoxicity of a naturally occurring cross-linking reagent for biological tissue fixation. J Biomater Sci Polym Ed 1999, 10:63-78.

25. Chau DY, Collighan RJ, Verderio EA, Addy VL, Griffin M: The cellular response to transglutaminase-cross-linked collagen. Biomaterials 2005, 26:6518-6529.

26. Jurgensen K, Aeschlimann D, Cavin V, Genge M, Hunziker EB: A new biological glue for cartilage-cartilage interfaces: tissue transglutaminase. J Bone Joint Surg Am 1997, 79:185-193.

27. Chen RN, Ho HO, Sheu MT: Characterization of collagen matrices crosslinked using microbial transglutaminase. Biomaterials 2005, 26:4229-4235.

28. Farndale RW, Buttle DJ, Barrett AJ: Improved quantitation and discrimination of sulphated glycosaminoglycans by use of 
dimethylmethylene blue. Biochim Biophys Acta 1986, 883:173-177.

29. Woessner JF: The determination of hydroxyproline in tissue and protein samples containing small proportions of this imino acid. Arch Biochem Biophys 1961, 93:440-447.

30. O'Brien J, Wilson I, Orton T, Pognan F: Investigation of the Alamar Blue (resazurin) fluorescent dye for the assessment of mammalian cell cytotoxicity. Eur J Biochem 2000, 267:5421-5426.

31. Ahsan T, Harwood F, McGowan KB, Amiel D, Sah RL: Kinetics of collagen crosslinking in adult bovine articular cartilage. Osteoarthritis Cartilage 2005, 13:709-715.

32. Sung HW, Huang DM, Chang WH, Huang RN, Hsu JC: Evaluation of gelatin hydrogel crosslinked with various crosslinking agents as bioadhesives: in vitro study. J Biomed Mater Res 1999, 46:520-530.

33. Sung HW, Chang Y, Liang IL, Chang WH, Chen YC: Fixation of biological tissues with a naturally occurring crosslinking agent: fixation rate and effects of $\mathrm{pH}$, temperature, and initial fixative concentration. J Biomed Mater Res 2000, 52:77-87.

34. Sung HW, Liang IL, Chen CN, Huang RN, Liang HF: Stability of a biological tissue fixed with a naturally occurring crosslinking agent (genipin). J Biomed Mater Res 2001, 55:538-546.

35. Gough JE, Scotchford CA, Downes S: Cytotoxicity of glutaraldehyde crosslinked collagen/poly(vinyl alcohol) films is by the mechanism of apoptosis. I Biomed Mater Res 2002, 61:121-130.

36. Huang LL, Sung HW, Tsai CC, Huang DM: Biocompatibility study of a biological tissue fixed with a naturally occurring crosslinking reagent. J Biomed Mater Res 1998, 42:568-576.

37. Chau DY, Collighan RJ, Verderio EA, Addy VL, Griffin M: The cellular response to transglutaminase-cross-linked collagen. Biomaterials 2005, 26:6518-6529.

38. Kaplonyi G, Zimmerman I, Frenyo AD, Farkas T, Nemes G: The use of fibrin adhesive in the repair of chondral and osteochondral injuries. Injury 1988, 19:267-272.

39. Homminga GN, Bulstra SK, Bouwmeester PS, van der Linden AJ: Perichondral grafting for cartilage lesions of the knee. J Bone Joint Surg Br 1990, 72:1003-1007.

40. Homminga GN, Buma $P$, Koot HW, van der Kraan PM, van den Berg WB: Chondrocyte behavior in fibrin glue in vitro. Acta Orthop Scand 1993, 64:441-445.

41. van Susante JL, Buma P, Schuman L, Homminga GN, van den Berg WB, Veth RP: Resurfacing potential of heterologous chondrocytes suspended in fibrin glue in large full-thickness defects of femoral articular cartilage: an experimental study in the goat. Biomaterials 1999, 20:1167-1175.

42. Wilson DJ, Chenery DH, Bowring HK, Wilson K, Turner R, Maughan J, West PJ, Ansell CW: Physical and biological properties of a novel siloxane adhesive for soft tissue applications. J Biomater Sci Polym Ed 2005, 16:449-472.

43. Williamson AK, Chen AC, Sah RL: Compressive properties and function-composition relationships of developing bovine articular cartilage. J Orthop Res 2001, 19:1113-1121.

44. Williamson AK, Chen AC, Masuda K, Thonar EJ, Sah RL: Tensile mechanical properties of bovine articular cartilage: variations with growth and relationships to collagen network components. J Orthop Res 2003, 21:872-880.

45. Williamson AK, Masuda K, Thonar EJ, Sah RL: Growth of immature articular cartilage in vitro: correlated variation in tensile biomechanical and collagen network properties. Tissue Eng 2003, 9:625-634.

46. DeGroot J, Verzijl N, Bank RA, Lafeber FP, Bijlsma JW, TeKoppele $\mathrm{JM}$ : Age-related decrease in proteoglycan synthesis of human articular chondrocytes: the role of nonenzymatic glycation. Arthritis Rheum 1999, 42:1003-1009.

47. DeGroot J, Verzijl N, Budde M, Bijlsma JW, Lafeber FP, TeKoppele $\mathrm{JM}$ : Accumulation of advanced glycation end products decreases collagen turnover by bovine chondrocytes. Exp Cell Res 2001, 266:303-310.

48. Chen AC, Temple MM, Ng DM, Verzijl N, DeGroot J, TeKoppele JM, Sah RL: Induction of advanced glycation end products and alterations of the tensile properties of articular cartilage. Arthritis Rheum 2002, 46:3212-3217. 